\title{
Coexistence of adenomyosis and endometrioid endometrial cancer: Role in surgical guidance and prognosis estimation
}

\author{
SALVATORE GIZZO $^{1}$, TITO SILVIO PATRELLI ${ }^{2}$, ANDREA DALL'ASTA ${ }^{2}$, STEFANIA DI GANGI ${ }^{1}$, \\ GIOVANNA GIORDANO ${ }^{2}$, COSTANZA MIGLIAVACCA ${ }^{2}$, MICHELA MONICA ${ }^{2}$, CARLA MERISIO ${ }^{2}$, \\ GIOVANNI BATTISTA NARDELLI ${ }^{1}$, MICHELA QUARANTA ${ }^{3}$, MARCO NOVENTA ${ }^{1}$ and ROBERTO BERRETTA ${ }^{2}$ \\ ${ }^{1}$ Department of Women's and Children's Health, Complex Operative Unit of Gynecology and Obstetrics, University of Padua, \\ Padua 35128; ${ }^{2}$ Department of Surgical Sciences, Complex Operative Unit of Gynecology and Obstetrics, \\ University of Parma, Parma 43125; ${ }^{3}$ Department of Obstetrics and Gynecology, \\ Complex Operative Unit of Gynecology and Obstetrics, University of Verona, Verona 37121, Italy
}

Received October 9, 2014; Accepted July 7, 2015

DOI: 10.3892/ol.2015.4032

\begin{abstract}
The aim of the current study was to diagnose the concomitant presence of adenomyosis (AM) in endometrioid endometrial cancer (EEC) in order to evaluate its value as an oncological prognostic marker. A retrospective analysis of 289 patients diagnosed with EEC who underwent total hysterectomy, bilateral salpingo-oophorectomy and pelvic-lymphadenectomy was conducted. The total cohort included 37 patients in Group A (those with concomitant AM and EEC) and 252 patients in Group B (those affected only by EEC). The following factors were evaluated: Presence or absence of AM, tumor grade, depth of myometrial invasion, tumor size, lymphovascular space involvement, lymph node status, peritoneal cytology, concomitant detection of endometrial atypical-hyperplasia or polypoid endometrial features and tumor stage according to the International Federation of Gynecology and Obstetrics (FIGO) classification. Uterine examination of different sections of uterine cervix, corpus, myomas and cervical or endometrial polyps was performed. The diagnosis of AM was confirmed when the distance between the lower border of the endometrium and the foci of the endometrial glands and stroma was $>2.5 \mathrm{~mm}$. Parametric and nonparametric statistical tests were performed when possible; continuous variables were analyzed using a Student's $t$-test, and categorical variables were analyzed by the $\chi^{2}$ test or Fisher's exact test. The association between FIGO stage and group was determined to be significant: $83.8 \%$ of Group A patients were categorized as FIGO stage I, vs. $68.7 \%$ of Group B patients. In addition, Group A was associated with lower grades in FIGO stage, myometrial invasion,
\end{abstract}

Correspondence to: Professor Salvatore Gizzo, Department of Women's and Children's Health, Complex Operative Unit of Gynecology and Obstetrics, University of Padua, 3 Via Giustiniani, Padua 35128, Italy

E-mail: ginecologia_padova@libero.it

Key words: adenomyosis, endometrioid endometrial cancer, prognostic factors, intraoperative estimating risk lymphovascular space involvement, lymph node involvement and tumor size. The findings suggest that the intraoperative evaluation of the presence of AM in patients with EEC may aid surgeons in estimating oncological risk and in selecting the most appropriate surgical treatment.

\section{Introduction}

Endometrial cancer (EC) is the most common malignant neoplasm of the female reproductive tract in developed countries (1). The disease is confined to the uterus in $>75 \%$ of cases and is typically characterized by a good prognosis, with an overall 5-year survival rate of $75-80 \%(2,3)$.

The most commonly diagnosed histological subtype is endometrioid endometrial cancer (EEC), which is characterized by a lower aggressiveness and a higher long-term disease-free survival following adequate primary and adjuvant treatment compared with that of other histological types of EC (2).

The evaluation of tumor grade, depth of myometrial invasion (MI), lymphovascular space involvement (LVSI), lymph node status (LNS) and peritoneal cytology is considered mandatory in order to assess the accuracy of the primary surgical staging and the necessity of adjuvant treatment and to determine the oncological prognosis $(4,5)$. Upon diagnosis of EC, pathologists focus on precisely evaluating the histological/morphological features of the disease: This evaluation has led to interest in the role of adenomyosis (AM) when occurring in association with EC.

$\mathrm{AM}$ is a benign condition defined as the presence of endometrial glands and stroma located $\geq 1$ intermediate power field away from the native endomyometrial junction $(6,7)$; notably, AM has been documented to coexist with EEC in 16-34\% of hysterectomy specimens obtained for the treatment of EC (8-14).

The significance of the presence of AM in estimating the prognosis of EEC is still debated, despite the fact that studies have reported an excellent prognosis for EEC with concomitant AM due to the lower histological grade and superficial MI detected in such cases (8-12). Neoplastic areas close to the 
foci of AM typically have a smooth contour and are frequently surrounded by endometrial stroma with or without benign endometrial glands (6). However, the predominant disagreement with regard to the significance of AM in EEC concerns whether it actively promotes or interferes with the MI of EC (13).

More recent studies have hypothesized that, in cases of EEC, the presence of AM may confer a poorer prognosis as it may be considered a precursor of an EEC lesion and an enabling factor allowing malignant cells to invade the myometrium by increasing the contact area (13-15).

The present study aimed to detect the coexistence of AM and EEC in order to evaluate whether AM may represent an oncological marker of favorable prognosis, associated with an earlier stage at diagnosis and biologically less aggressive behaviour.

\section{Materials and methods}

Patients. The present retrospective observational cohort study included patients referred to the Department of Surgical Sciences of the University of Parma (Parma, Italy) for surgical treatment of EC in the interval between January 1999 and January 2013. All postmenopausal patients diagnosed with EEC who underwent total hysterectomy (laparoscopic or laparotomic approach) with bilateral salpingo-oophorectomy, pelvic lymphadenectomy and peritoneal washing were considered eligible.

Patients with concurrent primary malignancies, EC of non-endometrioid histological type, previous use of hormone replacement therapy or raloxifene, levothyroxine treatment $(16,17)$, or a history of pelvic irradiation or intrauterine hysteroscopic surgery for metrorrhagia $(<1$ year prior to EEC diagnosis) (18) were excluded from the study.

For all patients, demographic data were documented, including age, body mass index (BMI), history of comorbid conditions (hypertension and/or diabetes) and previous use of tamoxifen $(19,20)$. All eligible patients were adequately informed at the time of admission regarding the possible use of their data for further analysis and publication in accordance with the Italian Privacy Law (675/96).

Uterine examination. All pathological reports were collected at the Pathological Anatomy and Histology Unit of the University of Parma following a specific uterine examination, conducted according to the guidelines of Rosai and Ackerman's Surgical Pathology (10th edition) (21), as follows. The uterus was opened by cutting with scissors through both lateral walls, from the cervix to the uterine cornua. The anterior half was marked (e.g. by cutting a small wedge on one side; note that if the tubes are attached, their insertion is anterior to that of the round ligament). Additional cuts were made through any large mass in the uterine wall. Parallel transverse sections were made through each half of the specimen, $\sim 1 \mathrm{~cm}$ apart, beginning at the upper level of the endocervical canal and ending short of completion at one side; each surface was carefully examined. Several sections of the cervix were made along the endocervical canal. At least one cross section was made for every myoma present and carefully examined, with additional cuts made for larger myomas as required.

The following sections were performed for histological evaluation: i) Cervix, one section from the anterior half and one from the posterior half; ii) corpus, $\geq 2$ sections taken close to the fundus and including the endometrium, a portion of myometrium and, thickness permitting, serosa; additional sections from any grossly abnormal areas; iii) myomas, 1-3 sections for myomas; sections from any grossly abnormal area (e.g. soft, fleshy, necrotic or cystic areas); iv) cervical or endometrial polyps, submitted whole unless extremely large.

For all patients, the diagnosis of AM was confirmed only when the distance between the lower border of the endometrium and the foci of endometrial glands and stroma exceeded $2.5 \mathrm{~mm}(22)$.

Other oncological features. In addition to the presence or absence of AM, the tumor grade, depth of MI, tumor size, LVSI, LNS, peritoneal cytology, concomitant detection of endometrial atypical hyperplasia or polypoid endometrial features, and tumor stage according to the International Federation of Gynecology and Obstetrics (FIGO) 2009 classification (5) were also recorded.

Aims of the present study. The primary aim was to compare the cases of EEC with concomitant AM (Group A) with the cases of EEC without AM (Group B) in terms of FIGO stage at diagnosis. The secondary aim was to compare Group A and Group B in terms of tumor size, LVSI, peritoneal cytology and the presence of endometrial atypical hyperplasia or polypoid endometrial features. Finally, the two groups were compared in terms of BMI, history of hypertension, diabetes and tamoxifen use.

Statistical analysis. Statistical analysis was performed using SPSS version 19 software for Windows (IBM SPSS, Armonk, NY, USA). The results were expressed as absolute numbers and percentages for discrete variables and as the mean \pm standard deviation for continuous variables. Appropriate parametric and nonparametric statistical tests were performed when possible, using the Kolmogorov-Smirnov test of normal distribution of the sample. Continuous variables were analyzed using a Student's $t$-test, and categorical variables were analyzed by the $\chi^{2}$ test or Fisher's exact test. $\mathrm{P}<0.05$ was considered to indicate statistical significance.

\section{Results}

General patient features. Among all patients who underwent surgery for EC during the recruitment interval, 289 cases were determined to be eligible for the current study. The general characteristics of all included patients are reported in detail in Table I. AM was detected upon histological investigation in only 37 patients (Group A), while the remaining 252 patients did not exhibit foci of AM (Group B).

Groups A and B were similar in terms of age at EEC diagnosis ( $66.6 \pm 11.7$ vs. $64.5 \pm 9.6$ years, respectively); however, the two groups (A vs. B) differed significantly with regard to mean BMI (31.9 \pm 3.8 vs. $27.3 \pm 4.4$ ), history of diabetes (75.7 vs. $32.1 \%$ ), hypertension (89.2 vs. $52 \%$ ) and tamoxifen intake (27 vs. $3.6 \%$ ) $(\mathrm{P}<0.001$; Fig. 1).

AM is associated with lower FIGO stage. Surgical FIGO stage was determined to differ significantly between the two study 
Table I. General features of patients in the two study groups.

\begin{tabular}{|c|c|c|c|c|}
\hline Variable & $\begin{array}{l}\text { All patients } \\
\quad(n=289)\end{array}$ & $\begin{array}{c}\text { Group A } \\
(n=37)\end{array}$ & $\begin{array}{l}\text { Group B } \\
(n=252)\end{array}$ & P-value \\
\hline Age at diagnosis, years & & & & $>0.05$ \\
\hline Mean \pm SD & $64.8 \pm 9.9$ & $66.6 \pm 11.7$ & $64.5 \pm 9.6$ & \\
\hline Range & $33-85$ & & & \\
\hline Body mass index & & & & $<0.001$ \\
\hline Mean $\pm \mathrm{SD}$ & $27.9 \pm 4.6$ & $31.9 \pm 3.8$ & $27.3 \pm 4.4$ & \\
\hline Range & $17-44$ & & & \\
\hline Diabetes, n (\%) & & & & $<0.001$ \\
\hline Yes & 109 (37.7) & $28(75.7)$ & $81(32.1)$ & \\
\hline No & $180(62.3)$ & $9(24.3)$ & $171(67.9)$ & \\
\hline Hypertension, n (\%) & & & & $<0.001$ \\
\hline Yes & $164(56.7)$ & $33(89.2)$ & $131(52.0)$ & \\
\hline No & $125(43.3)$ & $4(10.8)$ & $121(48.0)$ & \\
\hline Tamoxifen use, n (\%) & & & & $<0.001$ \\
\hline Yes & $19(6.6)$ & $10(27.0)$ & $9(3.6)$ & \\
\hline No & $270(93.4)$ & $27(73.0)$ & $243(96.4)$ & \\
\hline
\end{tabular}

Group A, adenomyosis present; Group B, adenomyosis absent; SD, standard deviation.

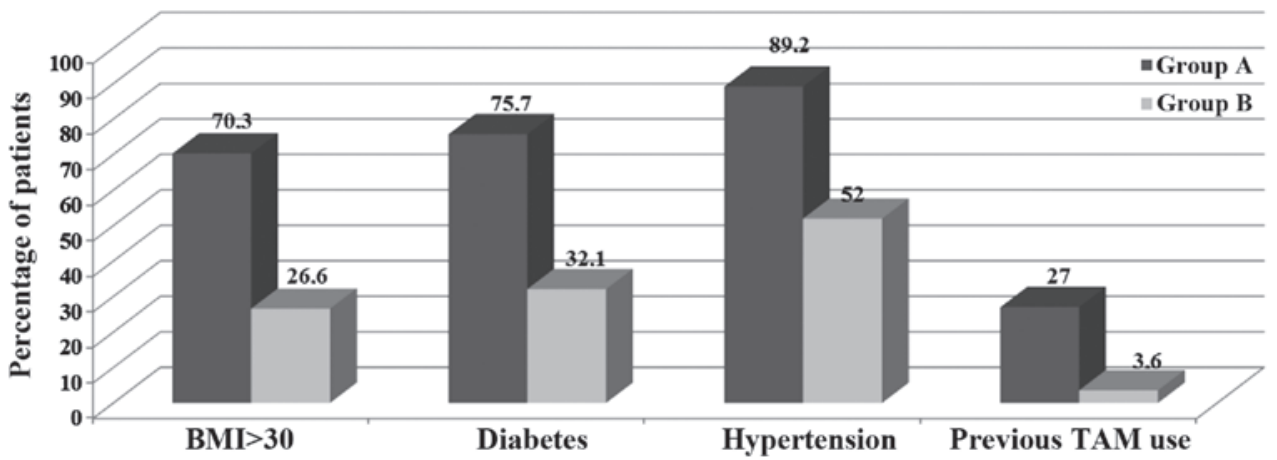

Figure 1. Percentage of patients with body mass index (BMI) >30, diabetes, hypertension and history of tamoxifen (TAM) use: Group A (adenomyosis present) vs. Group B (adenomyosis absent).

groups: $83.8 \%$ of Group A patients were assigned to FIGO stage I, vs. $68.7 \%$ of Group B patients $(\mathrm{P}<0.01)$. Notably, within FIGO stage I, $64.9 \%$ of Group A patients were categorized as stage IA, vs. $26.6 \%$ of Group B patients ( $<<0.001$; Fig. 2). A significant difference in FIGO stage between the two Groups was also confirmed in stage III: Stage IIIC1 was documented in $5.4 \%$ of Group A patients vs. $20.6 \%$ of Group B patients $(\mathrm{P}<0.001$; Fig. 2).

Surgical outcome. No significant difference was identified between the two groups in terms of the outcome of the surgical techniques used: The laparoscopic approach was performed in $21.6 \%$ of Group A vs. $19.8 \%$ of Group B. Similarly, the mean numbers of lymph nodes removed were $29.5 \pm 6.6$ and 28.7 \pm 7.4 , respectively ( $\mathrm{P}>0.05$; Table II).

Other oncological features. Although no difference was identified between Groups A and B in terms of positive peritoneal cytology rate (3.2 vs. $2.7 \%$, respectively), a borderline statistically significant difference was found in tumor grade $(\mathrm{G} 1$, 51.4 vs. $38.5 \%$; G2, $43.2 \%$ vs. $45.2 \%$; G3, $5.4 \%$ vs. $16.3 \%$, respectively; $\mathrm{P}=0.05)$. Furthermore, the two groups differed markedly in terms of MI: $<50 \%$ invasion of the uterus was detected in $70.3 \%$ of Group A vs. $31.7 \%$ of Group B patients $(\mathrm{P}<0.001)$; LVSI was positive in $10.8 \%$ of Group A vs. $40.1 \%$ of Group B $(\mathrm{P}<0.001)$, whilst a positive LNS was detected in $5.4 \%$ of Group A vs. $21 \%$ of Group B patients $(\mathrm{P}<0.05)$ (Table II; Fig. 3).

Histological features. The histological detection of atypical hyperplasia associated with EEC was reported in $29.7 \%$ of cases in Group A vs. 11.5\% in Group B ( $<<0.01)$, whilst the detection of concomitant endometrial polyps occurred in $37.8 \%$ of cases in Group A vs. $11.1 \%$ in Group B $(\mathrm{P}<0.001)$. The mean tumor sizes detected were $3.3 \pm 0.7 \mathrm{~mm}$ (Group A) vs. 4.1 $\pm 0.6 \mathrm{~mm}$ (Group B; P<0.001) (Table II; Fig. 4). 
Table II. Detailed surgical and histological features of patients in the two study groups.

\begin{tabular}{|c|c|c|c|c|}
\hline Variable & $\begin{array}{l}\text { All patients } \\
\quad(n=289)\end{array}$ & $\begin{array}{c}\text { Group A } \\
(n=37)\end{array}$ & $\begin{array}{l}\text { Group B } \\
(n=252)\end{array}$ & P-value \\
\hline Lymph nodes removed, $\mathrm{n}$ & & & & $>0.05$ \\
\hline Mean \pm SD & $28.8 \pm 7.3$ & $29.5 \pm 6.6$ & $28.7 \pm 7.4$ & \\
\hline Range & $16.1-41.9$ & & & \\
\hline Tumor size, mm & & & & $<0.001$ \\
\hline Mean \pm SD & $4.0 \pm 0.7$ & $3.3 \pm 0.7$ & $4.1 \pm 0.6$ & \\
\hline Range & $2.1-5.8$ & & & \\
\hline Endometrial hyperplasia, n (\%) & & & & $<0.01$ \\
\hline Yes & $40(13.8)$ & $11(29.7)$ & $29(11.5)$ & \\
\hline No & $249(86.2)$ & $26(70.3)$ & $223(88.5)$ & \\
\hline Endometrial polyps, $\mathrm{n}(\%)$ & & & & $<0.001$ \\
\hline Yes & $42(14.5)$ & $14(37.8)$ & $28(11.1)$ & \\
\hline No & $247(85.5)$ & $23(62.2)$ & $224(88.9)$ & \\
\hline Lymphovascular space involvement, n (\%) & & & & $<0.001$ \\
\hline Yes & $105(36.3)$ & $4(10.8)$ & $101(40.1)$ & \\
\hline No & $184(63.7)$ & $33(89.2)$ & $151(59.9)$ & \\
\hline Lymph node involvement, n (\%) & & & & $<0.05$ \\
\hline Yes & $55(19.0)$ & $2(5.4)$ & $53(21.0)$ & \\
\hline No & $234(81.0)$ & $35(94.6)$ & $199(79.0)$ & \\
\hline Myometrial involvement $<50 \%, \mathrm{n}(\%)$ & & & & $<0.001$ \\
\hline Yes & $106(36.7)$ & $26(70.3)$ & $80(31.7)$ & \\
\hline No & $183(63.3)$ & $11(29.7)$ & $172(68.3)$ & \\
\hline Positive peritoneal cytology, n (\%) & & & & $>0.05$ \\
\hline Yes & $9(3.1)$ & $1(2.7)$ & $8(3.2)$ & \\
\hline No & $280(96.9)$ & $36(97.3)$ & $244(96.8)$ & \\
\hline Tumor grading, $\mathrm{n}(\%)$ & & & & $=0.05$ \\
\hline G1 & $116(40.1)$ & $19(51.4)$ & $97(38.5)$ & \\
\hline $\mathrm{G} 2$ & $130(45.0)$ & $16(43.2)$ & $114(45.2)$ & \\
\hline G3 & $43(14.9)$ & $2(5.4)$ & $41(16.3)$ & \\
\hline Surgical approach, n (\%) & & & & $>0.05$ \\
\hline Laparoscopy & $58(20.1)$ & 8 (21.6) & $50(19.8)$ & \\
\hline Laparotomy & 231 (79.9) & $29(78.4)$ & $202(80.2)$ & \\
\hline
\end{tabular}

Group A, adenomyosis present; Group B, adenomyosis absent; SD, standard deviation.

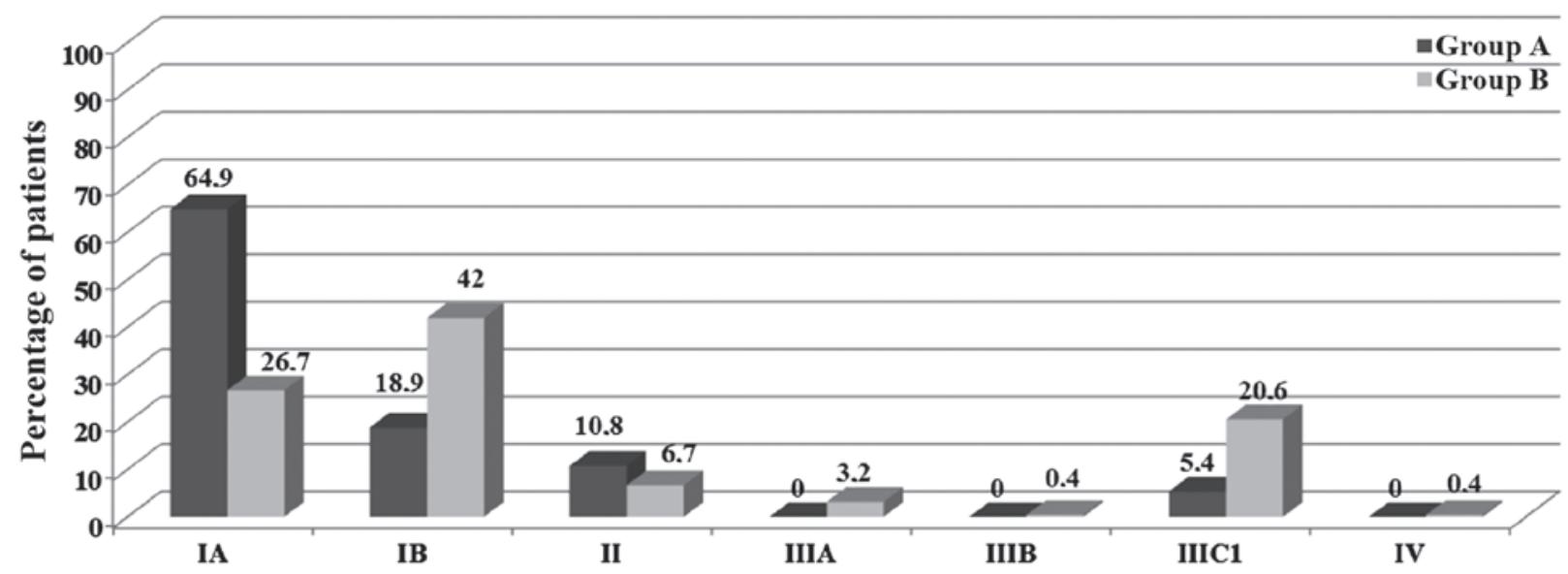

Figure 2. Correlation between FIGO stage and adenomyosis: Group A (adenomyosis present) vs. Group B (adenomyosis absent). 


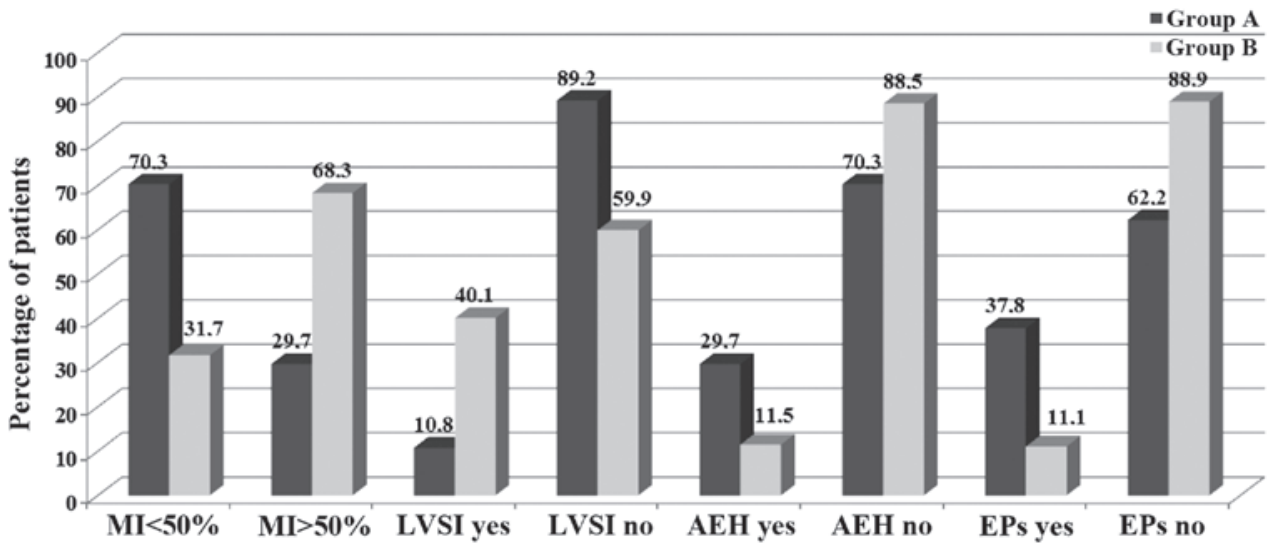

Figure 3. Differences in depth of myometrial invasion (MI), detection of lymphovascular space involvement (LVSI), detection of atypical endometrial hyperplasia (AEH) and detection of endometrial polyps (EPs): Group A (adenomyosis present) vs. Group B (adenomyosis absent).

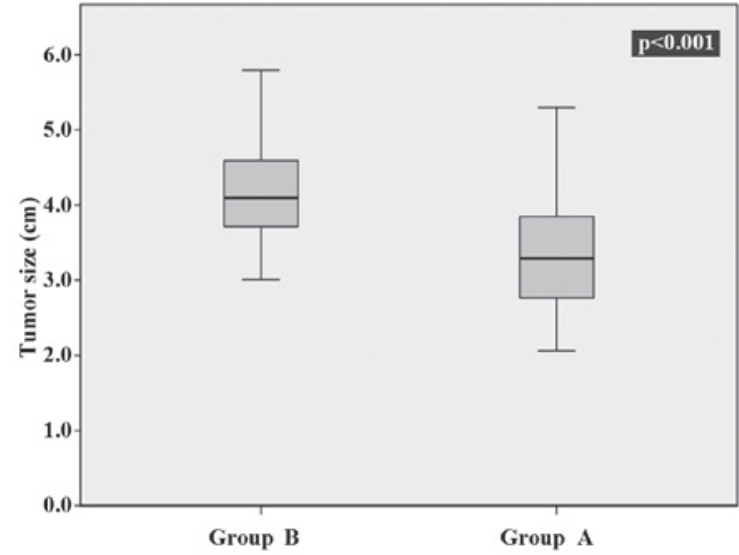

Figure 4. Correlation between primary tumor size and adenomyosis: Group B (adenomyosis absent) vs. Group A (adenomyosis present).

\section{Discussion}

EC is the most frequent gynaecological neoplasm in developed countries (23), with EEC the most frequent histological type. EEC is typically associated with a lower aggressiveness and an improved long-term disease-free survival following adequate treatment compared with that of other ECs (24).

Histology, depth of MI, tumor grade, presence of LVSI, positive peritoneal cytology, tumor size and LNS have been universally recognized as prognostic factors for determining long-term clinical course of the disease $(25,26)$. Although certain studies have evaluated the association between AM and the clinical course of EEC, the mechanism by which AM interferes with tumoral progression remains unresolved $(9,12,27,28)$.

$\mathrm{AM}$ is a common benign condition that is frequently detected in hysterectomy specimens containing EEC. However comprehending the relationship between EEC and AM remains an important challenge for gynaecologists and oncologists, as the role of $\mathrm{AM}$ in the pathogenesis and consequent clinical behaviour of EEC is unclear. Despite evidence of a frequent association between AM and EEC, which typically presents with a lower grade and lower degree of MI and lymph node involvement (4), no studies have been able to elucidate the relationship between these events $(9,12,27,28)$.
The frequent coexistence of the two diseases may be explained by a possible common risk factor or by a mutual pathogenic mechanism. Three theories have been proposed to describe the aetiology of AM: The first theory assumes that AM may originate from an invagination of the endometrial mucosa between bundles of uterine smooth muscle cells; the second theory suggests that endometrial tissue may enter the myometrium via the intramyometrial lymphatic system; and the third theory attributes AM to a de novo metaplasia arising from ectopic intramyometrial endometrial tissue (4).

In the present study, the prevalence of AM in specimens from hysterectomies performed for the treatment of EEC was determined to be $12.8 \%$, which was lower compared with the percentages reported by previous studies (8).

According to the current data, AM was strongly associated with the following risk factors: Personal history of diabetes, hypertension, high BMI and tamoxifen intake. Notably, these represent the same factors contributing to atypical hyperplasia and endometrial polyps through the creation of a hyper-estrogenic status. An overexpression of estrogen receptors in AM foci has been suggested as evidence of this possible association $(8,29)$ supported by the fact that the concomitant EEC diagnosed was frequently of type I, typical of a hyper-estrogenic status and endometrial hyperplasia $(3,30)$.

In 2007, Ismiil et al (13) suggested that the probability of deep MI is greater when AM is coexistent with grade 1 EEC, contrary to what may be expected (typically grade 1 EEC has a lower aggressiveness). The findings of the current study, in accordance with those of Musa et al (4), indicated that concurrent AM and EEC was associated with a lower tumor grade, MI of $<50 \%$, and the absence of LVSI and lymph node metastasis. This evidence explains and supports the strong inverse correlation between presence of AM and FIGO stage identified in the study: FIGO stage IA was detected in $64.9 \%$ of the cases with concomitant AM and in only $26.6 \%$ of cases without AM. Further confirmation of this trend is provided by the comparison of cases diagnosed at an advanced FIGO stage, as only $4 \%$ of all stage IIIC1 cases were positive for AM detection.

A strength of the present data is the homogeneity between the two groups in terms of lymph node removal, as all patients underwent lymphadenectomy and no significant difference was found in the mean number of lymph nodes removed 
in each group. Additionally, the current study confirms the importance of LVSI in predicting lymph node involvement ( $\sim 50 \%$ of patients with LVSI were of stage IIIC1), as well as demonstrating that the presence of AM represents a protective factor for LVSI (positive LVSI was detected at a rate of $\sim 10 \%$ in the presence of AM vs. $\sim 40 \%$ in the absence of AM).

Although this evidence has been reported by Musa et al (4), further studies are necessary in order to validate the process of detection of AM in intraoperative frozen sections and to assess its possible role as indicator of whether lymphadenectomy should be performed (19,31-33).

The rationale for a surgical evaluation of the coexistence of AM and EEC is comparable to that of intraoperatively defining tumor stage. Both could be used as a guideline for estimating the risk of lymph node involvement.

Numerous studies have demonstrated that, with an increase of tumor size, the probability of MI and detection of a higher tumor grade increases $(34,35)$. Based on this evidence, the current study analyzed the possible association between the presence of AM and the mean tumor size detected. Notably, a significant correlation between presence of AM and smaller tumor size was identified. To the best of our knowledge, this finding is the first confirmation that concurrent EEC and AM is associated with a higher differentiation grade and a lower tumor size. Lower tumor size is also often associated with a reduced MI, and the current findings confirm this: EEC concomitant with AM was associated with a statistically significant percentage of cases with $\mathrm{MI}<50 \%$. If confirmed by future studies, this evidence could clarify the mechanisms involved in LVSI and MI, as tumor size and grade are both independent risk factors for a poor oncological prognosis.

The inverse correlation between MI and AM may be explained by a possible altered adhesion mechanism between AM foci and cancer cells (with a reduced ability to go deeper into the myometrium) or by a lower cancer aggressiveness when AM coexists.

On the basis of these data, it may be speculated that the only disadvantage linked to coexistence of AM with EEC may be the increased difficulty in preoperative sonographic evaluation of the endometrium-myometrium junction and estimation of MI (36-38). The impossibility to evaluate the aforementioned feature due to the retrospective design represents a limitation of the current study. Additional study limitations include the absence of a preoperative Human Epididymis Protein 4 serum assay (39) and a long-term follow-up able to evaluate the impact of $\mathrm{AM}$ and disease recurrence rate/disease-free survival rate. Finally, the reason associated with a possible underestimation of AM detection rate (inferior to that reported in the literature) may be related to the exclusion of EC types other than EEC. We believe that a possible overestimation of LVSI was related to the fact that the pathologist was not blinded to the aim of the study.

In conclusion, in patients with EEC, the presence of associated AM may allow early detection with a good probability of diagnosing a disease with a higher grade of differentiation, a lower MI, an absence of LVSI, a small tumor size and a negative lymph node status. While an attempt at preoperative staging through ultrasound estimation of MI may be impaired by the presence of AM, the intraoperative evaluation of AM (in coexistence with EEC) may potentially aid the surgeon in estimating the oncological risk of the patient and selecting the most appropriate surgical treatment. Certainly, prospective large-scale studies are necessary to validate AM as an independent favorable oncological prognostic factor for EEC patients and to evaluate its usefulness, alone or in combination with other known prognostic factors, so as to define the most adequate personalized primary and adjuvant therapy.

\section{References}

1. Siegel RL, Miller KD and Jemal A: Cancer statistics, 2015. CA Cancer J Clin 65: 5-29, 2015.

2. Prat J: Prognostic parameters of endometrial carcinoma. Hum Pathol 35: 649-462, 2004.

3. Giordano G, D'Adda T, Bottarelli L, Lombardi M, Brigati F, Berretta R and Merisio C: Two cases of low-grade endometriod carcinoma associated with undifferentiated carcinoma of the uterus (dedifferentiated carcinoma): A molecular study. Pathol Oncol Res 18: 523-528, 2012.

4. Musa F, Frey MK, Im HB, Chekmareva M, Ellenson LH and Holcomb K: Does the presence of adenomyosis and lymphovascular space invasion affect lymph node status in patients with endometrioid adenocarcinoma of the endometrium? Am J Obstet Gynecol 207: 417 e1-e6, 2012.

5. Pecorelli S: Revised FIGO staging for carcinoma of the vulva, cervix and endometrium. Int J Gynaecol Obstet 105: 103-104, 2009.

6. Hanley KZ, Dustin SM, Stoler MH and Atkins KA: The significance of tumor involved adenomyosis in otherwise low-stage endometrioid adenocarcinoma. Int J Gynecol Pathol 29: 445-451, 2010

7. Garcia L and Isaacson K: Adenomyosis: Review of the literature. J Minim Invasive Gynecol 18: 428-437, 2011.

8. Bergeron C, Amant F and Ferenczy A: Pathology and physiopathology of adenomyosis. Best Pract Res Clin Obstet Gynaecol 20: 511-21, 2006.

9. Mittal KR and Barwick KW: Endometrial adenocarcinoma involving adenomyosis without true myometrial invasion is characterized by frequent preceding estrogen therapy, low histologic grades and excellent prognosis. Gynecol Oncol 49: 197-201, 1993.

10. Jacques SM and Lawrence WD: Endometrial adenocarcinoma with variable-level myometrial involvement limited to adenomyosis: A clinicopathologic study of 23 cases. Gynecol Oncol 37: 401-407, 1990.

11. Hernandez E and Woodruff JD: Endometrial adenocarcinoma arising in adenomyosis. Am J Obstet Gynecol 138: 827-832, 1980.

12. Kucera E, Hejda V, Dankovcik R, Valha P, Dudas M and Feyereisl J: Malignant changes in adenomyosis in patients with endometrioid adenocarcinoma. Eur J Gynaecol Oncol 32: 182-184, 2011.

13. Ismiil N, Rasty G, Ghorab Z, Nofech-Mozes S, Bernardini M, Ackerman I, Thomas G, Covens A and Khalifa MA: Adenomyosis involved by endometrial adenocarcinoma is a significant risk factor for deep myometrial invasion. Ann Diagn Pathol 11: 252-257, 2007.

14. Seidman JD and Kjerulff KH: Pathologic findings from the Maryland women's health study: Practice patterns in the diagnosis of adenomyosis. Int J Gynecol Pathol 15: 217-221, 1996.

15. Ismiil ND, Rasty G, Ghorab Z, Nofech-Mozes S, Bernardini M, Thomas G, Ackerman I, Covens A and Khalifa MA: Adenomyosis is associated with myometrial invasion by FIGO 1 endometrial adenocarcinoma. Int J Gynecol Pathol 26: 278-283, 2007.

16. SaccardiC, Gizzo S, Ludwig K, Guido M, Scarton M, Gangemi M, Tinelli R and Litta PS: Endometrial polyps in women affected by levothyroxine-treated hypothyroidism - histological features, immunohistochemical findings and possible explanation of etiopathogenic mechanism: A pilot study. Biomed Res Int 2013: 503419, 2013.

17. Gizzo S, Saccardi C, Patrelli TS, et al: Update on raloxifene: Mechanism of action, clinical efficacy, adverse effects and contraindications. Obstet Gynecol Surv 68: 467-481, 2013.

18. Saccardi C, Conte L, Fabris A, et al: Hysteroscopic enucleation in toto of submucous type 2 myomas: Long-term follow-up in women affected by menorrhagia. J Minim Invasive Gynecol 21: 426-430, 2014 
19. Gizzo S, Di Gangi S, Bertocco A, Noventa M, Fagherazzi S, Ancona E, Saccardi C, Patrelli TS, D'Antona D and Nardelli GB: Levonorgestrel intrauterine system in adjuvant tamoxifen treatment: Balance of breast risks and endometrial benefits - systematic review of literature. Reprod Sci 21: 423-431, 2014.

20. Saccardi C, Gizzo S, Patrelli TS, Ancona E, Anis O, Di Gangi S, Vacilotto A, D'Antona D and Nardelli GB: Endometrial surveillance in tamoxifen users: Role, timing and accuracy of hysteroscopic investigation: Observational longitudinal cohort study. Endocr Relat Cancer 20: 455-462, 2013.

21. Rosai J (ed): Female reproductive system. In: Rosai and Ackerman's Surgical Pathology. Vol 2. 10th edition. Mosby Elsevier, pp1399-1422, 2011.

22. Kurman RJ, Ellenson LH and Ronnett BM (eds): Blaustein's Pathology of the Female Genital Tract. 6th edition. Springer, New York, 2011.

23. Wright JD, Barrena Medel NI, Sehouli J, Fujiwara K and Herzog TJ: Contemporary management of endometrial cancer Lancet 379: 1352-1360, 2012.

24. Berretta R, Patrelli TS, Faioli R, Mautone D, Gizzo S, Mezzogiorno A, Giordano G and Modena AB: Dedifferentiated endometrial cancer: An atypical case diagnosed from cerebellar and adrenal metastasis: Case presentation and review of literature. Int J Clin Exp Pathol 6: 1652-1657, 2013.

25. Gizzo S, Fabris A, Litta P and Saccardi C: Estimated intermediate risk endometrial cancer: Debate and new perspectives on therapy individualization and prognosis establishment starting from a peculiar case. Int J Clin Exp Pathol 7: 2664-2669, 2014.

26. Berretta R, Patrelli TS, Migliavacca C, Rolla M, Franchi L, Monica M, Modena AB and Gizzo S: Assessment of tumor size as a useful marker for the surgical staging of endometrial cancer. Oncol Rep 31: 2407-2412, 2014.

27. Chrysostomou M, Akalestos G, Kallistros S, Papadimitriou V, Nazar S and Chronis G: Incidence of adenomyosis uteri in a Greek population. Acta Obstet Gynecol Scand 70: 441-444, 1991.

28. Gün I, Oner O, Bodur S, Ozdamar O and Atay V: Is adenomyosis associated with the risk of endometrial cancer? Med Glas (Zenica) 9: 268-272, 2012.

29. Ueki K, Kumagai K, Yamashita H, Li ZL, Ueki M and Otsuki Y: Expression of apoptosis-related proteins in adenomyotic uteri treated with danazol and GnRH agonists. Int J Gynecol Pathol 23: 248-258, 2004.
30. Merisio C, Berretta R, De Ioris A, Pultrone DC, Rolla M, Giordano G, Tateo S and Melpignano M: Endometrial cancer in patients with preoperative diagnosis of atypical endometrial hyperplasia. Eur J Obstet Gynecol Reprod Biol 122: $107-111,2005$.

31. Kim YB and Niloff JM: Endometrial carcinoma: Analysis of recurrence in patients treated with a strategy minimizing lymph node sampling and radiation therapy. Obstet Gynecol 82: 175-180, 1993.

32. Todo Y, Kato H, Kaneuchi M, Watari H, Takeda M and Sakuragi N: Survival effect of para-aortic lymphadenectomy in endometrial cancer (SEPAL study): A retrospective cohort analysis. Lancet 375: 1165-1172, 2010.

33. Patrelli TS, Berretta R, Rolla M, Vandi F, Capobianco G, Gramellini D, Bacchi Modena A and Nardelli GB: Pelvic lymphadenectomy in endometrial cancer: Our current experience. Eur J Gynaecol Oncol 30: 536-538, 2009.

34. Mariani A, Webb MJ, Keeney GL and Podratz KC: Routes of lymphatic spread: A study of 112 consecutive patients with endometrial cancer. Gynecol Oncol 81: 100-104, 2001.

35. Schink JC, Rademaker AW, Miller DS and Lurain JR: Tumor size in endometrial cancer. Cancer 67: 2791-2794, 1991.

36. Boes AS, Tousseyn T, Vandenput I, Timmerman D, Vergote I, Moerman P and Amant F: Pitfall in the diagnosis of endometrial cancer: Case report of an endometrioid adenocarcinoma arising from uterine adenomyosis. Eur J Gynaecol Oncol 32: 431-434, 2011.

37. Berretta R, Merisio C, Piantelli G, Rolla M, Giordano G, Melpignano $\mathrm{M}$ and Nardelli GB: Preoperative transvaginal ultrasonography and intraoperative gross examination for assessing myometrial invasion by endometrial cancer. J Ultrasound Med 27: 349-355, 2008.

38. Savelli L, Ceccarini M, Ludovisi M, Fruscella E, De Iaco PA, Salizzoni E, Mabrouk M, Manfredi R, Testa AC and Ferrandina G: Preoperative local staging of endometrial cancer: Transvaginal sonography vs. magnetic resonance imaging. Ultrasound Obstet Gynecol 31: 560-566, 2008.

39. Gizzo S, Ancona E, Saccardi C, D'Antona D, Nardelli GB and Plebani M: Could kidney glomerular filtration impairment represent the 'Achilles heel' of HE4 serum marker? A possible further implication. Clin Chem Lab Med 52: e45-e46, 2014. 